Slavica

bruxellensia

\section{Slavica bruxellensia}

Revue polyphonique de littérature, culture et histoire

slaves

9 | 2013

Érotisme

\title{
La mannequination : un procédé moderne d'érotisme chez Bruno Schulz
}

Les Boutiques de cannelle et Le Sanatorium au croque-mort

\section{Jessy Neau}

\section{(2) OpenEdition}

Journals

Édition électronique

URL : http://journals.openedition.org/slavica/1362

DOI : 10.4000/slavica.1362

ISSN : 2034-6395

Éditeur

Université libre de Bruxelles - ULB

Référence électronique

Jessy Neau, "La mannequination : un procédé moderne d'érotisme chez Bruno Schulz », Slavica bruxellensia [En ligne], 9 | 2013, mis en ligne le 15 avril 2013, consulté le 25 mai 2020. URL : http:// journals.openedition.org/slavica/1362; DOI : https://doi.org/10.4000/slavica.1362

Ce document a été généré automatiquement le 25 mai 2020

\section{(c) (†) $\odot$}

Les contenus de Slavica bruxellensia sont mis à disposition selon les termes de la Licence Creative Commons Attribution - Pas d'Utilisation Commerciale - Pas de Modification 3.0 France. 


\title{
La mannequination: un procédé moderne d'érotisme chez Bruno Schulz
}

Les Boutiques de cannelle et Le Sanatorium au croque-mort

\author{
Jessy Neau
}

\section{Introduction}

1 L'œuvre littéraire de l'écrivain juif d'expression polonaise Bruno Schulz (1892-1942) est principalement constituée de deux recueils de nouvelles: Sklepy cynamonowe [Les Boutiques de cannelle ${ }^{1}, 1934$ ] et Sanatorium pod klepsydra [Le Sanatorium au croque-mort ${ }^{2}$, 1937]. Tous les récits possèdent entre eux une certaine continuité : il s'agit, selon l'écrivain lui-même ${ }^{3}$, de mythifier sa ville natale de Drohobycz - ville située en Pologne avant la Deuxième Guerre mondiale, actuellement en Ukraine - dans laquelle la figure $\mathrm{du}$ père s'élève au-dessus des autres personnages qui reviennent d'une nouvelle à l'autre. Sous l'impulsion créatrice de cette figure paternelle, les personnages du quotidien se retrouvent métamorphosés en créatures étranges, évoluant sur des chorégraphies parfois animales, parfois mécanisées ${ }^{4}$.

2 Un certain nombre de récits de Schulz mettent en scène un processus éminemment moderne d'érotisation : celui d'une mécanisation du corps. Automates dans la nouvelle «Le printemps », infirmières zombiesques dans «Le Sanatorium au croque-mort » et surtout les mannequins des quatre récits que l'on trouve dans Les Boutiques de cannelle: toutes ces créatures sont l'objet de la fascination du héros, Joseph, alter ego de Schulz. En littérature, le corps mécanisé trouve à s'exprimer dans la figure de l'automate, dont les deux caractéristiques principales sont l'anthropomorphisme ainsi que la capacité de mouvement autonome. ${ }^{5}$ La figure de l'automate connaît une grande fortune dans la littérature fantastique, fascination initiée par E.T.A. Hoffmann dans «L'homme au sable $»^{6}$, ce dernier récit mettant en scène les amours de Nathanaël et de la poupée Olympia. Le rapport entre désir érotique et corps mécanisé est très prégnant dans la 
littérature fantastique qui se plait à figurer ce qui relève du simulacre et du dédoublement du sujet. ${ }^{7}$

3 L'érotisme fantastique de Schulz est spécifique à plus d'un titre, car il emprunte à plusieurs traditions littéraires que nous proposons de comparer. Si le motif du mannequin ou de l'automate s'inscrit dans l'héritage germanique du XIX siècle, l'articulation générale entre érotisme et fantastique s'ancre chez Schulz dans une sensibilité proche des Russes du même siècle, celle de Nikolaj V. GogoL' ou de Fëdor M. Dostoevskij, en ce que la figuration du désir libidinal est diluée dans une contamination par l'objet amoureux de l'espace et du temps. Notre étude se veut ainsi d'abord lier l'écriture érotique de Schulz à son héritage russe. En prenant pour exemple le début de Nevskij prospekt (La Perspective Nevski) de GogoL', nous montrerons comment la prééminence de l'expression amoureuse tient de sa relation labyrinthique à l'espace, et comment sa saisie métaphorique la fait tendre vers le paroxysme.

4 L'écriture fortement érotisée de Schulz se constitue principalement autour de cette dynamique paroxystique, phénomène qui, on le verra, dépasse la présence thématique de ces figures mécanisées. D'abord parce qu'elle est un procédé éminemment réflexif, renvoyant au sujet qui contemple l'objet l'image de son propre désir. Mais également par la vocation des mannequins à contaminer les êtres et les objets, qui se mettent à devenir eux aussi des créatures indifférenciées. Cette contamination n'est ainsi pas seulement de l'ordre du paroxysme, mais également de celui de la déviance, par la prolifération des créatures féminines qui tendent à esquisser des séries et des blocs, notion que l'on emprunte ici à Gilles Deleuze et Félix Guattari dans leur lecture de Franz Kafka, dont la sensibilité est à rapprocher de celle de Schulz.

5 Paroxysme et déviance sont les deux pôles de l'érotisme chez Schulz, définis par la réalisation littérale de l'eros dans la littérature fantastique.

\section{L'érotisme fantastique slave}

6 La littérature fantastique affectionne particulièrement les motifs érotiques et se plait à faire de la figuration surnaturelle ou étrange le lieu d'une forte charge sexuelle. Selon l'essai de Camille Dumoulié sur le fantastique, plusieurs arguments peuvent ainsi rentrer en ligne de compte pour dégager la faculté privilégiée du genre fantastique à mettre en scène l'érotisme ${ }^{8}$.

7 Les manifestations extrêmes de l'amour fantastique seraient les plus propres à manifester la nature et les effets de l'amour, de la même manière que la psychanalyse freudienne développe une vision de l'amour uniquement par l'étude de ses bizarreries et déviances. La passion amoureuse a «la force de supprimer les refoulements et de rétablir les perversions ${ }^{9}$.

8 En outre, une étrange similarité est à l'œuvre entre la syntaxe du fantasme et la logique du genre fantastique : le fantasme et le fantastique possèdent tous deux la « visée de la réalisation d'une forme, manifestée de façon transgressive $»^{10}$.

9 Le retour du même propre au fantastique, par ses jeux de miroirs, de dédoublements ou par ses figures de revenants, manifestent un retour à l'inanimé dû au caractère infernal de la répétition. L'autre pôle de la relation entre le fantasme et le fantastique a trait à l'investissement narcissique de la libido. L'autre comme double narcissique témoigne 
de la nature toujours ambivalente du désir : il est l'objet d'un investissement valorisant, mais qui peut aussi révéler par effet de miroir le vide du sujet et la vacuité de son désir.

Les textes fantastiques sont, plus peut-être que les textes mimétiques, perméables à une mise en scène qui autorise des figurations de l'indicible et que le désir, on le sait depuis Jacques Lacan, est articulé à un manque. « Nous retenons principalement de ces arguments la nature à la fois thématique - une réalisation littérale de phénomènes et processus figurés - et paroxystique du genre fantastique quant à l'érotisme. $»^{11}$

11 Cependant, une spécificité de l'écriture érotique dans les nouvelles fantastiques russes du grand siècle est, contrairement à ce qui a cours dans les nouvelles des autres littératures européennes à la même époque, l'absence remarquable de motifs clairement identifiables comme figurations du désir libidinal.

Si les récits fantastiques de Prosper Mérimée, Théophile Gautier ou Hoffmann penchent du côté de la thématisation du désir en mettant en scène portraits, statues, animations d'objets ou créatures diaboliques féminines, l'érotisme fantastique des personnages masculins chez Gogol' et Dostoevskij se cristallise essentiellement sur le motif de la rencontre amoureuse.

Il nous paraît pertinent de lier cet érotisme à l'une de ses variantes modernes. Passer de Gogo' à Schulz n'implique pas uniquement un saut temporel considérable. C'est également un profond bouleversement quant aux modalités du fantastique que révèle ce choix. D'abord, parce que l'onirisme de l'écriture de Schulz appartient bien plus à un fantastique de l'étrangeté qu'à un fantastique plus classique tel que celui de Gogol'. Largement comparé à Kafka, Schulz s'attache notamment à mythifier la réalité et à faire de tout évènement quotidien un processus démiurgique.

D'autre part, l'expression de la sexualité chez Schulz s'ancre dans des motifs concrets, principalement les mannequins, alors que nous avions précédemment bien souligné l'absence de figure concrète de l'érotisme chez Gogol'. Cependant, une même dynamique de projection du désir sur l'espace et le temps est à l'œuvre chez les deux auteurs, une dynamique qui fonde la tonalité fantastique au-delà de la prééminence accordée à un motif surnaturel.

Dans La Perspective Nevski de GogoL', "l'hésitation " ${ }^{12}$ fantastique naît de l'apparition d'une jeune femme, que le personnage de Piskariov - jeune peintre timoré - va se mettre à suivre dans les rues de Saint-Pétersbourg. D'une qualification matérialiste et sociale de la jeune femme aperçue ( C'est probablement une dame de la haute société, continua-t-il en soupirant. Son manteau à lui seul vaut plus de quatre-vingt roubles!» ${ }^{13)}$, l'on est amené progressivement à envisager une dématérialisation de "l'inconnue » à mesure que Piskariov la suit :

16 Tout l'ouvrage de Dumoulié ${ }^{16}$ lie le schéma de brisure temporelle induit par la rencontre amoureuse à la brisure de l'autre temps fantastique ainsi qu'à la forme particulière de la nouvelle.

17 Mais l'expression paroxystique de la rencontre amoureuse dans La Perspective Nevski va en réalité au-delà de la simple exagération de l'effet irréel donné par l'apparition féminine. En effet, l'écriture de GogoL', de façon caractéristique, ne fait pas qu'influer sur le temps fantastique mais aussi sur l'espace, contaminé par l'apparition.

18 Un moment de la nouvelle "Le Sanatorium au croque-mort » de Schulz peut éclairer ces différentes tensions à l'œuvre dans l'érotisme schulzien. Il s'agit du moment où Joseph reçoit un colis, contenant un ouvrage pornographique [SpK, 180-182]. Son père, 
mécontent, le somme d'aller le consulter dans l'arrière-boutique. En ouvrant le paquet, Joseph s'aperçoit qu'il ne s'agit pas d'un livre, mais d'un télescope. Celui-ci a le pouvoir de déformer la réalité, d'en faire voir les facettes les plus intimes et obscures; en outre, un étrange coup du sort fait se métamorphoser la lunette en chenille.

Le désir transgressif opère ainsi une même translation dans l'espace, la chenille parcourant ensuite un voyage loufoque dans le sanatorium, et possède le pouvoir de transformer l'environnement de Joseph qui voit alors les commis et boutiquiers évoluer dans des chorégraphies saugrenues. Cette dynamique de transformation alchimique et transgressive par le désir se retrouve également dans la nouvelle "Le printemps", dans laquelle la fascination de Joseph pour le personnage de Bianca engendre la métamorphose du temps ordinaire en temps cosmique, un temps de l'horoscope que la métaphore du " treizième mois ${ }^{19}$ surdétermine. L'espace s'opacifie également :

C'est que, au-delà de la différence entre les motifs, les paramètres de l'expression transgressive du désir s'oriente chez Schulz, d'une manière assez similaire à celle de Gogo', vers les mêmes procédés de mise en crise spatio-temporelle ainsi que par la brèche constante que la métaphore se plait à surdéterminer :

21 Ainsi, les procédés d'érotisation chez Schulz reposent sur la même présence paradoxale du fantastique que chez GogoL' : jouant de l'ambiguïté métaphorique et des registres sensoriels bien plus que sur la présence concrète d'un motif surnaturel. Mettant en œuvre une indétermination quant à l'appartenance claire de ces textes au genre fantastique, ils n'en sont pas moins porteurs de cette tendance à pousser le désir le plus loin possible dans son expression paroxystique. Si nous envisageons ainsi l'étude de l'érotisme de Schulz à travers une figure bien concrète, celle du mannequin, notre posture tend néanmoins à la saisir dans sa valeur emblématique, comme symbole d'une sexualité masochiste et déviante, ainsi que comme point d'ancrage d'une éthique de la création démiurgique.

\section{Les mannequins et la création}

Schulz souligne dans sa présentation des Boutiques de cannelle ${ }^{20}$ la continuité thématique entre tous ses récits: tous ont pour cadre la province galicienne du village de Drohobycz. Les mêmes personnages reviennent: Adèle, la bonne délurée, la mère, Bianca, Rodolphe ou Chloma, camarades de Joseph ainsi que les commis. Les Boutiques de cannelle ne sont pas tout à fait un recueil de nouvelles, ni tout à fait un roman : les récits sont à la fois reliés et indépendants.

Nous soulignons cette homogénéité diégétique afin de justifier les passages et correspondances entre différents récits de Schulz que nous réalisons dans cette étude. Toute référence à un récit particulier de Schulz ne peut donc être considérée comme valable uniquement pour elle-même: c'est d'ailleurs bien ce que l'adaptation cinématographique réalisée par Wojciech Has en 1973 du Sanatorium au croque-mort montre, à savoir l'impossibilité de n'adapter qu'un seul récit de l'écrivain polonais, dont tous les récits s'interpénètrent en permanence. Pour Anne Guérin-Castell, il s'agit d'un " parcours non linéaire à travers les nouvelles de Schulz »"

Envisageons d'abord les composantes érotiques de la figure du mannequin dans les Boutiques de cannelle. Celles-ci reposent sur deux modalités principales: la banalité et l'artifice. La figure de mannequin est amenée par les deux couturières que le père, 
Jacob, a embauchées pour réaliser des finitions sur des costumes : "Portée par elles, une dame silencieuse entrait dans la pièce, créature de toile et d'étoupe avec une boule de bois noir en guise de tête. $»^{24}$

L'apparition de cette "dame silencieuse " s'inscrit d'abord dans le vertige d'une découverte de l'existence de simulacre, dont nous allons éclairer la valeur érotique. Le mannequin apporté par les deux couturières est " une idole sans voix », " maîtresse de la situation »; elle est tout autant convoitée qu'une femme diminuée, imitant la femme réelle mais nécessairement amoindrie. Deux fonctions que possèdent les simulacres de l'être humain sont identifiées dans l'ouvrage Visages de double par Pierre Jourde et Paolo Tortonese : «Tout ce qui imite les formes du corps humain finit par acquérir soit un sens de révélation, soit de parodie de l'être humain. ${ }^{25}$

Le mannequin chez Schulz a bien, en effet, un sens de révélation, et ce parce qu'elle est une figure spéculaire. Figure de l'immobilité, passive mais contemplatrice, elle renvoie à celui qui l'observe le reflet de sa propre immobilité et contemplation : «Sans bouger, elle surveillait en silence le travail des jeunes filles. Elle accueillait d'un air critique et désobligeant leurs efforts pour lui plaire lorsqu'elles s'agenouillaient devant elle en essayant des morceaux de drap faufilés de blanc. ${ }^{26}$

L'amour de la banalité a trait au néant, étant issu d'un pur jeu de forme sans visée apparente. Ce sont les diatribes du père qui tendent vers cette célébration du vide : «Moins de fond, plus de forme! Ah! Comme une diminution du fond allègerait le monde ! » ${ }^{27}$

À partir de ce moment, Joseph affirme la nécessité de livrer le message paternel sur la création, celle-ci prenant pour point de départ cette figure de mannequin. La première fonction révélatrice du mannequin a donc lieu dans le développement d'une doctrine métaphysique de la matière seconde.

Il s'agit d'une doctrine "plus qu'hétérodoxe ${ }^{28}$ qui se développe dans les nouvelles suivantes. Nous résumerons celle-ci comme étant celle de la "genèse seconde ", que le titre de la nouvelle suivante, "Traité des Mannequins ou la seconde genèse ", indique. Celle-ci se réalise en plusieurs moments. Le père réalise d'abord une apologie du meurtre nécessaire en vertu de l'absence de mort absolue et de l'expérimentation sur les formes de la matière ${ }^{29}$. S'ensuit dès lors un long programme posant comme base la volonté de réaliser une deuxième genèse, qui créerait les êtres à l'imitation des mannequins. La «Fin du traité des mannequins» [SC, 73-80] réalise l'apogée de la tension démiurgique en insufflant à toute matière, à tout objet une vitalité absolue, mais dont l'aboutissement n'aurait lieu que par un devenir-simulacre. Il faut souligner au passage que chez Schulz, les prophéties et conférences du père tournent généralement autour de cette métaphysique du simulacre et de la pseudo-matière. ${ }^{30}$

Ce qui nous intéresse le plus et que nous rattachons à l'érotisme de Schulz concerne bien entendu l'aspect de pacotille. L'ensemble des Mannequins est, dans les Boutiques de cannelle, généralement analysé par l'aspect métaphysique qui s'en dégage. La nature premièrement érotique de cet ensemble, qui nous paraît pourtant fondamentale, est ainsi rarement évoquée.

31 En effet, l'expression de cette volonté créatrice provient bien du père, et non de Joseph : il s'agit des récits dans lesquels Jacob s'exprime le plus. Or, il est à noter qu'une dynamique inverse entre les différentes voix de ces récits est à l'œuvre. Plus Jacob célèbre la vitalité et la singularité de la matière, plus les êtres présents sous l'œil du 
narrateur tendent à se désincarner et à céder au néant. Les deux couturières, Pauline et Poldine, sont ainsi de plus en plus immobiles, spectatrices passives des conférences du père.

De la sorte, une deuxième fonction de révélation a également cours avec la figure du mannequin, celle-ci ayant davantage à voir avec la sexualité proprement dite. L'érotisme de Schulz est souvent abordé du point de vue du masochisme de l'écrivain. Selon Sigmund Freud, le masochisme a trait à la passivité, et à ce titre est une perversion éminemment féminine. Or, Schulz lui-même souligne le caractère proprement féminin de l'attitude du mannequin: "Le moloch était inexorable comme seuls peuvent l'être les molochs féminins - et les renvoyait sans cesse à leur travail. $»^{31}$

S'il nous faut d'abord noter l'aspect misogyne de cette conception, qui s'ancre dans un discours conscient de l'imminence de la fin d'un ordre patriarcal judéo-chrétien, c'est que la dimension réflexive de cette posture est fondamentale. La nature spéculaire de la figure du mannequin affecte les couturières. Mais ne peut-on pas, en filigrane, percevoir cette réflexivité comme réfractant la propre attitude de Joseph, soumis non seulement à la divinité immobile du mannequin mais également à celle du père écrasant dans sa posture démiurgique?

C'est ce que l'attrait de Joseph pour l'indifférenciation et même la tendance à une désintégration semble indiquer, puisqu'elle abolit toute singularité. De ce point de vue, la banalité semble bien correspondre à une forme de masochisme, puisque celui-ci tend toujours, pour la psychanalyse, vers la destruction et le retour à l'inanimé. Freud fait du masochisme une introjection du sadisme originaire, devenant «un témoin et un vestige de cette phase de formation dans laquelle se produisit cet alliage si important pour la vie, de la pulsion de mort et de la pulsion de vie. $»^{32}$

Tout comme chez Kafka, le héros éprouve à la fois une fascination infantile pour ce père mythifié, tout en éprouvant de plus en plus son déclin imminent - à l'image de la décrépitude de Jacob, métamorphosé en cafard ${ }^{33}$. Une dynamique qu'il n'est pas difficile de relier à l'intuition d'un monde archaïque ayant entamé sa décadence, celui de l'Empire austro-hongrois et de la société patriarcale dans laquelle Joseph évolue au milieu des drapiers et marchands de Drohobycz. C'est bien ce que montre la nouvelle "Le printemps", mettant en scène des automates créés à partir de grandes figures autoritaires comme Maximilien d'Autriche [SpK, 103].

De prime abord, se dessinerait chez Schulz une opposition binaire en ce qui concerne les simulacres : celle de parodie, essentiellement ancrée dans ces figures d'automates, et celle de fascination, donnée par les mannequins. En réalité, comme la nature ambivalente du masochisme le sous-tend déjà, chaque figure anthropomorphe est prise dans une tension constante, entre un débordement et un évidement.

\section{La mannequination}

37 Car si la pulsion de mort est acquise par la banalité et l'indifférenciation, il n'en est pas moins que l'ensemble des mannequins dans les Boutiques de cannelle est l'occasion de rendre compte une nouvelle fois chez Schulz du débordement de la matière et de la tentation démiurgique de toute création. Il faut souligner la nature fort originale de cette tension extrême entre débordement et évidement, même si celle-ci constitue un 
paradigme du simulacre : «L'imitation peut se résoudre dans l'excès (multiplication des corps, pouvoirs exceptionnels qui leur sont attribués) ou bien dans l'épuisement (corps vidés, inertes, etc.) $»^{34}$

C'est surtout le déplacement des qualités généralement attribuées aux mannequins qui constitue l'indice d'un excès - la prolifération des mannequins. C'est avec la mannequination qu'apparaît une forme d'ironie, une vocation parodique de l'érotisme.

Ce déplacement se fait sur les deux couturières, Pauline et Poldine, desquelles Joseph et son père admirent « la structure de leur banale beauté $»^{35}$. Indifférenciées, affublées de noms à consonances semblables, le duo est comme mannequiné par l'hommage que le père fait à leur pure forme.

L'artificialité et la banalité de la «dame silencieuse » se déplacent dans les femmes réelles, qui deviennent sous l'impulsion démiurgique du père, des créatures, au sens d'une véritable création de l'homme dont elles sont l'objet, comme le titre de la nouvelle suivante, « Traité des mannequins ou la seconde Genèse » l'indique.

41 C'est d'abord dans la passivité et l'immobilité déjà évoquées que l'extension du caractère de mannequin se réalise sur les deux couturières. Le début des «Mannequins » les saisissait dans le mouvement presque orchestré de leur travail avec le tissu: «Les jeunes filles, sans y prêter attention, foulaient aux pieds ces débris d'un carnaval possible, ces hardes d'une mascarade avortée. Elles s'ébrouaient avec un rire nerveux pour faire tomber les bouts de tissu, caressaient des yeux les miroirs. ${ }^{36}$ "

2 Mais à mesure que le père est dit rentrer dans les profondeurs les plus " hérésiarques " de sa conférence, Pauline et Poldine sont de plus en plus silencieuses, devenant 《immobiles $»^{37}$ et à la fin presque incapables du moindre mouvement, étant " figées sur leurs chaises (...) [et] étrangement engourdies ${ }^{38}$.

3 En réalité, des indices de leur réification étaient d'emblée donnés par le narrateur. Leur portrait physique s'apparente curieusement à la description de matières artificielles : «(...) l'émail de leurs yeux brillait comme le vernis de leurs souliers et les boucles des jarretelles sous leurs robes retroussées par le vent. $»^{39}$

4 À la fin, les qualifications employées par le narrateur au sujet des deux couturières font s'allier sa propre perception de leur transformation en créatures figées avec les propos du père sur la seconde genèse. Ainsi, «les yeux vitreux» de Pauline et Poldine sont l'expression pour Joseph de ce que son père appelle la création: "Les jeunes filles restaient fascinées, les yeux vitreux. À la vue de leurs visages qu'une attention soutenue avait tendus et stupéfiés, à la vue de leurs joues enfiévrées, l'on aurait pu se demander si elles relevaient de la première ou de la seconde Création. $»^{40}$

4 À partir de là, la voix de Joseph semble se faire l'écho de la doctrine paternelle, la réalisant dans son univers de Drohobycz. Les visages stupéfiés des couturières renvoient non seulement à l'absence d'expression du mannequin apporté au début de la nouvelle, mais soulignent également la fascination que le spectateur ne manque pas d'éprouver en écoutant la doctrine hétérodoxe de Jacob. Autrement dit, la mannequination renvoie au moi de Joseph, voire au moi du lecteur, aux prises avec les tumultes de la pensée métaphysique du père.

46 La mannequination ne peut dès lors épargner les autres personnages des Boutiques de cannelle. Agissant par contamination, le texte de Schulz réalise à la lettre l'adresse du père faite à son public: "Figures de musée Grévin, mes chères demoiselles - 
commençait-il, mannequins de foire, oui ; mais même sous cette forme, gardez-vous de les traiter à la légère. $»^{41}$

Dès lors, même le personnage féminin de l'univers schulzien le plus déluré, et le seul tenant tête à Jacob - Adèle, la bonne - se retrouve à la fin de l'ensemble mannequiné, immobile et stupéfié par la seule intervention du mot "mannequin». Mais cette mannequination ultime révèle ici encore sa nature toute réflexive. En devenant mannequin, Adèle force le père à se figer à son tour, frappé d'impuissance et de mutisme :

La contamination semble donc totale. Elle va par ailleurs se réaliser dans d'autres nouvelles de Schulz. Ainsi des infirmières aux pas mécanisés de la nouvelle «Le Sanatorium au croque-mort ", dans laquelle le temps régulier, le temps à intervalles, fuit dans les personnages devenus incarnations du temps séculaire. ${ }^{43}$

Outre le goût de Schulz pour la pacotille et un certain masochisme envers la femme toujours dominante, inaccessible et artificielle, l'extension du caractère de mannequin à la femme réelle opère une manière de renvoi à toute création, à toute réalité physique : «Une poupée peut signifier la beauté féminine, mais aussi la triste et banale matérialité du corps, la mécanique de l'organisme: les articulations du mannequin rappellent à l'homme que lui aussi est fait de pièces. $»^{44}$

Créatures différentes des mannequins, les automates de la nouvelle «Le printemps » sont des figures de cire qui répliquent des personnages ayant existé, Dreyfus, Garibaldi ou Bismarck. [SpK, 103]

51 La créature d'automate est ainsi liée, inversement aux mannequins passifs, à l'action héroïque, à l'histoire devenue légende. Mais l'action et l'histoire ont perdu leur signification, c'est bien ce que cette prolifération semble signifier :

Les figures de cire sont des simulacres liés à une perte. Non seulement elles sont des figures de la prolifération, mais elles prolifèrent aussi dans la nouvelle « Le Sanatorium au croque-mort ", par l'affirmation à plusieurs reprises du caractère de simulacre que l'existence du père malade a pris :

53 Le simulacre ou "succédané " existentiel est ainsi au cœur de toute une réflexion globale menée à diverses reprises chez Schulz, une notion que celui-ci développe luimême dans son aspect métaphysique. L'essence des choses ne s'acquiert que par médiations constantes, artifices et décalages :

54 Il semble que dans cette célébration de la matière ironique et dans une esthétique qui tend vers le carnaval, l'indifférenciation entre les êtres de chair et les êtres de cire dépasse le désir amoureux pour ne plus mettre en scène que des figures désincarnées, non plus exclusivement féminines. Pourtant, il nous faut voir que cette dynamique paradoxale entre excès et évidement institue bel et bien un monde érotique singulier chez Schulz.

\section{L'eros féminin démiurgique}

Afin de relier l'érotisme qui a cours dans l'œuvre de Schulz avec une éthique et une pensée qui lui seraient contemporaines dans la même aire culturelle, il nous faut ici revenir à la relation qu'entretient la figure du mannequin avec le père dans l'œuvre de Schulz. 

l'œuvre de Schulz, par un renversement du classique complexe d'œEdipe. La phobie de la femme est ainsi liée à la fascination du père, cette figure toute-puissante substituée à l'œEdipe maternel. Le bureau du père dans Le Sanatorium au croque-mort devient le lieu de toute initiation. Le débordement et l'excès n'est ainsi pas tant celui des mannequins que celui du père: omniprésent, tout-puissant, mais également saisi dans des parallélismes constants avec les situations que vit Joseph. Ainsi, les figures féminines ne peuvent être que figées ou vides, à l'instar de nos mannequins.

La sexualité dans l'œuvre de Schulz met bien en scène une dégradation de la femme en tant que matière. Artur Sandauer évoque ainsi une manière "organique ${ }^{47}$ avec laquelle l'écrivain de Drohobycz décrit les personnages féminins. Il souligne ainsi le caractère parfaitement diabolique de cette « dégradation ", qui tend à enfermer le sujet désirant dans la monomanie et dans un enfer masochiste sans possibilité d'interaction avec l'autre.

La réification de la femme réalise le goût généralisé pour la pacotille, que le père érige en doctrine: «Le Démiurge était amoureux de matériaux solides, compliqués et raffinés : nous donnons, nous, la préférence à la camelote. Nous sommes attirés et positivement séduits par la camelote, par tout ce qui est vulgaire et quelconque. $»^{48}$

Mais l'enfermement dans le masochisme n'est pas la seule conclusion à tirer de cet amour de la pacotille. Comme Sandauer le souligne, il y a là également une fascination de la part de Schulz pour une modernité en train de se réaliser. Dans la nouvelle « La rue des crocodiles", Joseph est ainsi subjugué par la vulgarité du "pseudoaméricanisme » des boutiques, le caractère artificiel et corrompu du décor, frappé de stupeur devant les vendeuses posant pour faire la démonstration des articles aux clients. Les mannequins peuvent donc marquer le signe d'une entrée dans la consommation moderne, dans une ère d'artefacts et de produits consommables. Pour Sandauer, l'érotisme de Schulz est coloré historiquement : le péché sent la naphtaline des articles vendus dans les magasins modernes, le sexe prend une valeur marchande.

Les mannequins de Schulz nous font donc voir les prémices de la société actuelle, dont la sexualité est à la fois plus que présente, les corps parfaits s'étalant partout, et en même temps banalisant cette sexualité qui ne possède plus tant de charge érotique que renvoyant le sujet du désir à sa propre solitude et à l'autodestruction irrémédiable que son désir ne manque pas d'engendrer. A ce titre, la fin de "La rue des crocodiles" suggère bien un aspect différent, mais parallèle, de cet érotisme moderne :

61 Les proliférations d'images ici évoquées - que l'on retrouvera dans la nouvelle «Jojo » $[\mathrm{SpK}, 208-211]^{49}$ - donnent à voir un érotisme de simulacre plus prosaïque, moins allégorique que celui donné par les mannequins, mais qui possède la même caractéristique de ne tendre que vers la stérile représentation de lui-même et sur sa nature intrinsèque de simulacre.

Enfin, la modernité de l'érotisme schulzien se lie, par les mannequins, à la figure démiurgique du père. Or, cette relation est en réalité paradoxale : certes, c'est bien le père qui proclame une tentative démiurgique de créer les mannequins. Mais comme nous l'avons déjà esquissé, les voix du père et du fils ne s'entremêlent pas tout à fait uniformément dans cet ensemble de récits. A y regarder de plus près, les mannequinations envisagées par Joseph vont bien davantage dans le sens de l'évidement 
et de la dégradation de la matière que dans celui d'une re-création ou d'un élan vital accordé à toute matière, comme Jacob le prône.

Cette discordance des voix amène alors à s'interroger sur une éventuelle défaite du père dans l'œuvre de Schulz. La fin du récit «Traité des mannequins ou la seconde Genèse " va bien dans ce sens: le père est à son tour frappé d'immobilité, réduit temporairement à l'impuissance. Comme dans La métamorphose de Kafka, mais d'une manière inverse, le père sera transformé dans un autre récit en cafard ${ }^{50}$

Dès lors, la sexualité dirigée vers l'artefact et la camelote de Joseph peut être interprétée comme une mise à mort du monde commercial qu'incarne le père, celui d'avant la modernité, constitué par le négoce archaïque et l'artisanat.

Cependant, un dernier moment de notre étude doit s'attacher à considérer ce qui reste " après » les mannequins. La prolifération féminine n'est pas uniquement liée à la mannequination, et l'érotisme de Schulz s'épanouit également, certes de manière plus marginale qu'avec les figures de mannequins, mais de façon certaine, dans d'autres réagencements.

\section{Après la mannequination}

Dans Les Boutiques de cannelle et Le Sanatorium au croque-mort, Joseph rencontre sans cesse des figures féminines que l'on peut qualifier de professionnelles : la marchande de glaces ou les infirmières dans la nouvelle "Le Sanatorium au croque-mort», la vendeuse de tickets de cinéma dans « La nuit de juillet », la maitresse d'école dans « Le retraité ».

On retrouve ici la forme de « série » dégagée par Deleuze et Guattari chez Kafka :

Avec «La nuit de juillet», la prolifération atteint son point culminant, puisqu'elle féminise entièrement l'univers de Joseph. Sa sœur vient de mettre au monde un nouveau-né, et cette présence engendre le rattachement de la maison à toute une série d'évocations de lieux féminins fermés :

Avec un envahissement devenu total, nous pouvons poursuivre notre emprunt à Deleuze et Guattari : il n'y pas chez Kafka que des « séries ", mais aussi des «blocs » et des « intensités ». Ce que les deux auteurs attribuent à Kafka ne prétend pas s'appliquer littéralement à Schulz, même si, on l'aura compris, extrêmement nombreux sont les points communs entre les deux écrivains.

Le cinquième et dernier sens donné par Deleuze et Guattari à la notion de bloc chez Kafka peut nous intéresser en ce qu'elle met en jeu la force expressive («procédure d'expression et procédé de contenu») et travaillée d'une manière singulière, de l'enfance, par rapport au souvenir qui n'est pas de même dynamique :

71 Les connexions érotiques chez Schulz sont donc constituées d'un réseau bien plus complexe que celui seul des mannequins, tout en se nourrissant de leur pouvoir de contamination. Les séries féminines ont fortement à voir avec elles, tout en amenant le bloc d'enfance à reconstituer un désir originaire, matriarcal, que Helbig-Mischewski qualifie de salvateur : «Face à l'apocalypse qui achève Les Boutiques à la cannelle, c'est le sein d'Adèle qui porte l'espoir d'un recommencement. $\|^{55}$

On voit que de quelque nature que ces proliférations se réalisent, toutes atteignent au cœur de la problématique de l'engendrement qui réalise une abolition des forces 
masculines. Le fait que celui-ci signifie ou non une victoire du matriarcat n'est pas établi : mais toutes ces défaites rattachent la prolifération et les images de la diffraction à la contre-initiation permanente, à une transition culturelle faite d'instabilité entre deux mondes.

\section{Conclusion}

73 Le fantastique «classique » fait jouer la surdétermination d'objets surnaturels pour inscrire dans son projet un érotisme paroxystique, un désir nécessairement excessif. Le « hors-norme » de la sexualité devient, comme dans la psychanalyse, la norme.

En cela, Schulz est bien un auteur fantastique, mais davantage dans la tradition de Gogo' ou de Dostoevskij que dans celle d'Hoffmann, par le jeu ambivalent des métaphores et de la contamination. Chez ces auteurs, la métamorphose du sujet n'a lieu que par sa transfiguration réalisée par le désir. En effet, point d'objet magique, de double, de phénomène d'apparition clairement rattaché au surnaturel: ici, c'est uniquement la créature féminine qui engendre une métamorphose du sujet mais également des éléments environnants.

Schulz met en outre un pied dans la modernité en poussant bien plus loin le jeu métaphorique. L'analogie a en effet le pouvoir de contaminer tous les êtres, métamorphosant toutes les créatures en les frappant d'indifférenciation. Les êtres deviennent au contact des métaphores des séries, des catégories se situant quelque part entre le type et l'individu. L'érotisme de Schulz se fait alors conflictuel, oscillant constamment entre le désir de l'indifférenciation, la modernité des artefacts et la sexualité devenue produit de consommation d'un côté, et un rêve de retour au sein maternel et au terrifiant gynécée de l'autre.

Sexualité orientée vers la représentation pornographique, relation soumise au père, voyeurisme ; tentative par le biais de la métaphore de révéler les plus intimes recoins de l'univers et mécanisation du désir sont autant d'éléments de la sexualité schulzienne qui se dégagent de notre lecture, en étant toujours pris dans une correspondance nécessaire avec toute une doctrine plus générale de la création. Et autant de modalités érotiques inscrites dans ce que nous avons appelé la mannequination, procédé qui lie dans l'œuvre de l'écrivain de Drohobycz les différentes notions de fantastique, d'érotisme et de prolifération.

\section{NOTES}

1. Schulz B., Sklepy cynamonowe, Towarzystwo Wydawnicze Rój, 1934, Varsovie. Traduction française : Les Boutiques de cannelle, traduction de Thérèse Douchy, Georges Lisowski et Georges Sidre, Gallimard, Paris, 1992, Paris. A la demande de l'auteur de l'article, c'est la version française qui a été utilisée ici. Dans la suite de l'article, les citations seront marquées par [SC] suivi du numéro de page. 
2. Schulz B., Sanatorium pod KlepsydrĄ, Towarzystwo Wydawnicze Rój, 1937, Varsovie. Traduction française: Le Sanatorium au croque-mort, traduiction de Thérèse Douchy, Allan Kosko, Georges Sidre et Suzanne Arlet, Gallimard, Paris, 2010. A la demande de l'auteur de l'article, c'est la version française qui a été utilisée ici. Dans la suite de l'article, les citations seront marquées par [SpK] suivi du numéro de page.

3. Schulz B., «La mythification de la réalité», in: Euvres complètes, Denoël, Paris, 2004, pp. 185-187.

4. Nous signalons ici les ouvrages les plus importants concernant Bruno Schulz: Unmasking Schulz: New Combinations, Further Fragmentations, Ultimate Reintegrations (Schulz démasqué : nouvelles combinaisons, fragmentations ultérieures et réintégrations ultimes), sous la direction de De Bruyn D. \& Van Heuckelom K., Rodopi, Amsterdam, 2009 ; Bruno Schulz: nouvelles lectures, nouvelles significations / Bruno Schulz: New Readings, New Meanings, Proceedings of the International Conference, Montreal, Canada, 2007 ; Stownik schulzowski (Dictionnaire Schulz), sous la direction de Bolecki Wł., Jarz̨̨bski J. \& Rośko St., słowo/obraz terytoria, Gdańsk, 2003.

5. Tel que l'Encyclopédie définit l'automate. Définition reprise dans : Jourde P. \& Tortonese P., Visages du double : un thème littéraire, Nathan, Paris, 1996, p. 165.

6. Hoffmann E. T. A., Don Juan, suivi de Der Sandmann (L'Homme au sable), traduit par MichelFrançois Demet, LGF Livre de poche, Paris, 1991, pp. 75-188.

7. En 1947 déjà, un inventaire fut réalisé. Nous le citons à titre d'exemple : Chapuis A., Les automates dans les œuvres d'imagination, Editions du Griffon, Neufchâtel, 1947.

8. Dumoulié C., Cet obscur objet de désir. Essais sur les amours fantastiques, L'Harmattan, Paris, 1995, pp. 9-14.

9. Freud S., "Pour introduire le narcissisme», in: La vie sexuelle, PUF, Paris, p. 70, cité par Dumoulié C., op. cit., p.9.

10. Dumoulié C., op. cit., p.60.

11. Bozzetto R., "Corps et décors du désir ", in: Cahiers du GERF, n 5, Grenoble, 1998, pp. 121-132.

12. Au sens bien connu du terme, hérité de la définition désormais classique du fantastique, à savoir celle donnée par Tzvetan Todorov dans: Introduction à la littérature fantastique, Le Seuil, Paris, 1970.

13. GogoL' N. V., La Perspective Nevski (titre original : Nevskij prospekt), Librio, Paris, 1996, p.16.

14. Ibid., pp.21-22.

15. Dostoevskij F. M., Les Nuits blanches (titre original : Belye noČi), traduit par André Markowicz, Actes Sud, Paris, 1992.

16. Dumoulié C., op. cit. voir en particulier les pages 39 à 45 .

17. Gogol' N. V., op. cit., p. 22.

18. Idem.

19. «Les évènements que nous allons relater ont eu lieu ce treizième mois, superfétatoire en quelque sorte et postiche, sur la douzaine de pages vides de la grande chronique du calendrier. » Schulz B., « La nuit de la grande saison » [SpK, 129].

20. Schulz B., «Sur Les Boutiques de cannelle », in : CEuvres complètes, op. cit., p.201.

21. Guérin-Castell A., La place de Manuscrit trouvé à Saragosse dans l'œuvre cinématographique de Wojciech Jerzy Has, thèse de doctorat soutenue le 30 janvier 1998 à l'Université de Paris VIIIVincennes, p. 436 (non-publié).

22. Il s'agit de la traduction littérale du titre de Schulz, et non celui retenu par l'éditeur français.

23. Ibid., p. 436.

24. "Les mannequins", in : SC, 62.

25. Jourde P. \& Tortonese P., op. cit., p.163.

26. «Les mannequins", in : SC, 62.

27. Ibid., p.65. 
28. Ibid., p.66.

29. On pourrait voir ici une référence à Donatien Alphonse François de Sade ou à l'hédonisme. Cependant, les références à la démiurgie biblique placent cette apologie dans une parodie matérialiste de l'Ancien Testament, et acquièrent une valeur plus métaphysique.

30. «L'autre automne », SpK, 133-139.

31. «Les mannequins », SC, 63.

32. Freud S., «Le problème économique du masochisme » (1924), in CEuvres complètes, vol. XVII, PUF, 1992, pp. 9-23.

33. «La Morte-saison », in : SpK, 139-145.

34. Jourde P. \& Tortonese P., op. cit., p.163.

35. "Les mannequins », in : SC, 64.

36. Ibid., p.63.

37. "Traité des mannequins ou la seconde genèse ", in : SC, p.70.

38. «Fin du traité des mannequins ", in : SC, 75.

39. "Les mannequins", in : SC, 64.

40. "Traité des mannequins ou la seconde genèse ", in : SC, 70.

41. "Fin du traité des mannequins ", in : SC, 73

42. "Traité des mannequins ou la seconde genèse ", in : SC, 71-72.

43. «Le Sanatorium au croque-mort » met en scène un étrange sanatorium dans lequel les lois temporelles sont manipulées. Le temps y est itératif, circulaire, avec la possibilité de réactiver le passé.

44. Jourde P. \& Tortonese P., op. cit., p.163.

45. Schulz dans une lettre adressée à Stanisław Ignacy Witkiewicz datant de 1934, dans : Schulz B., Lettres perdues et retrouvées, avec neuf dessins de l'auteur, préface et traduction du polonais par Maria Craipeau, Pandora/Textes, Aix-en-Provence, 1979, p.54.

46. Helbig-Mischewski B., « Séduit par la mère : déchéance du patriarcat dans la prose de Bruno Schulz », extrait de la conférence Kartografia/e mniejszosci literackich $i$ innych (Cartographie(s) des littératures mineures et autres), 18 novembre 2004, Université Paris-IV Sorbonne. Traduction de K. Bessière. En ligne. URL : http ://www.helbig-mischewski.de/schulz03.pdf. Consultation : 15 avril 2013.

47. Sandauer A., «Rzeczywistość zdegradowana (Rzecz o Brunonie Schulzu)» (La réalité dégradée - à propos de Bruno Schulz) in : Zebrane pisma krytyczne (Ecrits critiques choisis), t. I, Państwowy Instytut Wydawniczy, Varsovie, 1991, pp. 557-580.

48. "Traité des mannequins ou la seconde genèse ", in : SC, 70.

49. Le personnage de Jojo a pour seule occupation de découper les articles de journaux les plus intéressants et de les coller ensuite dans un cahier, selon un ordre connu de lui seul.

50. «La Morte-saison », in : SpK, 139-145.

51. Helbig-Mischewski B., art. cit., p. 3.

52. Deleuze G. \& Guattari F., Kafka : pour une littérature mineure, Ed. de Minuit, Paris, 1975, p. 97.

53. Ibid., p. 114

54. Deleuze G. \& Guattari F., op. cit., p. 141

55. Helbig-Mischewski B., art. cit., p. 5. 


\section{RÉSUMÉS}

Un certain nombre de récits de Bruno Schulz mettent en scène un processus éminemment moderne d'érotisation: celui d'une mécanisation du corps. Automates dans la nouvelle «Le Printemps ", infirmières zombiesques dans «Le Sanatorium au croque-mort » et surtout les mannequins de la suite de nouvelles «Les mannequins» que l'on trouve dans Les Boutiques de cannelle : toutes ces créatures sont l'objet de la fascination du héros, Joseph. Lamannequination est donc d'abord un processus de prolifération et d'indifférenciation. Mais si l'obsession de la pacotille engendre une multiplication des figures artificielles, c'est en réalité surtout le déplacement des qualités généralement attribuées aux mannequins qui constitue l'érotisation maximale du corps cette fois exclusivement féminin. Les caractéristiques des mannequins se déplacent dans les femmes réelles, qui deviennent, sous l'impulsion de la figure du père démiurge, des créatures.Schulz achève ce processus en féminisant entièrement son univers, les femmes appartenant toutes à une forme de série. C'est donc dans une perspective de lier modernité et érotisme à l'aune d'une sexualité tournée vers l'objet, le fétiche et les simulacres, que notre étude se propose d'étudier les composantes de l'érotisme de Schulz, en effectuant des points de comparaison avec, d'une part, l'héritage fantastique slave dans lequel la figure spéculaire du mannequin peut s'inscrire et, d'autre part, avec certaines formes de mises en scènes déviantes du désir que l'on trouve chez Franz Kafka. Paroxysme et déviance sont ainsi les deux pôles de l'érotisme chez Schulz, définis par la réalisation littérale de l'eros dans la littérature fantastique.

\section{INDEX}

Index géographique : Pologne, Russie

Mots-clés : érotisme en littérature, littérature fantastique, littérature polonaise

oeuvretraite Schulz B. : Sanatorium pod KlepsydrĄ (Le Sanatorium au croque-mort), Schulz B. :

Sklepy cynamonowe (Les Boutiques de canelle)

Index chronologique : entre-deux-guerres, XXe siècle

\section{AUTEURS}

\section{JESSY NEAU}

Doctorante Etudes Françaises, University of Western Ontario (London, Canada) jneau@uwo.ca 